\title{
Asymmetric Cost Sharing Mechanisms
}

Eric J. Friedman

\author{
School of ORIE, Cornell University \\ Ithaca, NY, 14850 \\ ejf27@cornell.edu
}

\begin{abstract}
We describe the construction and analysis of asymmetric Cost Sharing mechanisms, in which a variety of axioms are applied to subsets of the agents/goods. We show that the analysis can be quite subtle as apparently similar axiomatizations lead to significantly different results; in particular, combinations of symmetric mechanisms can be extremely asymmetric and biased. In addition, we characterize some interesting mixed mechanisms.
\end{abstract}

Keywords:

\section{Introduction}

A standard definition of an a axiom is a "self evident truth." At first glance, this definition appears to motivate much of the literature on Cost Sharing. Most axiomatizations in the literature are based on "universal axiomatizations" where the same set of axioms are applied uniformly to all agents or goods.

For example, many of the classic works in this area arose from the characterization of the Shapley-Value [1] and the characterization of the AumannShapley Cost Sharing mechanism $[2,3,4]$ by such a set of axioms. Since these pioneering works there have been many axioms proposed to characterize a variety of Cost Sharing mechanisms. (See [5] for a review and bibliography.)

However, in many of these papers, the motivations for various axioms are based more on applications. For example, in the axiomatization of the Aumann-Shapley mechanism, the motivation that homogeneous goods should have equal prices is motivated by the more plebian concern that agents might be able to arbitrage the system. Even the motivation for Scale Invariance, which is based on the incomparability of different goods, need not apply 
when there is a natural basis for making comparisons of scale between select goods. More directly, the Demand Monotonicity axiom introduced by Moulin [6] and used in the axiomatization of the Friedman-Moulin mechanism [7] is motivated by the need to prevent agents from padding their demands to lower their total cost.

In this paper, we consider the use of axioms in an application dependent way. We will view axioms as constraints that need to be imposed in certain settings. For example, the axiomatization of the Aumann-Shapley mechanism arose from the sharing of telephone costs at Cornell University in the 1970s [8]. Consider a modern version of this setting, in which agents pay for use of various Information Technology services. If we assume that individual agents (such as students) are responsible only for a minuscule fraction of the calls and are able to use each others' accounts to access the services (such as students in a computer lab) then one should apply the equal prices axiom; however, for other users, the equal prices axiom is less compelling, such as for faculty in different departments and different physical locations. Next consider the case of a small group of large users whose demand is a significant fraction of the total demand. It is important that these large users not be encouraged to pad their demand, so one should require the Cost Sharing mechanism to be Demand Monotonic for them, but this axiom need not be applied to the students, since a single small agent cannot significantly affect prices.

Thus, we see the motivation for mixed axiomatizations of Cost Sharing mechanisms. In this paper we provide an initial analysis of these problems and focus on analyzing the situation described above. In so doing we reveal some surprising subtleties to the analysis and construct new families of Cost Sharing mechanisms. Although we focus on specific Cost Sharing mechanisms and axioms in this paper, we note that our methods are quite general and apply to a much broader class of Cost Sharing mechanisms and axioms than we directly analyze.

This paper is organized as follows. In the following section we present our basic model. Then in Section 3 we review the axiomatizations of several well known mechanisms. Section 4 considers the "high level axiomatizations" directly mixing mechanisms, while Section 5 considers the "lower level axiomatizations", mixing low level axioms, which turns out to be significantly stronger (more restrictive). In Section 6 we discuss some symmetry issues and conclude in Section 7 with a discussion. 


\section{Model}

We consider the standard Cost Sharing setting. There are a set of agents $N=\{1,2, \ldots, n\}$ and a vector of (bounded) demands

$$
q \in Q=\left\{q \in \Re^{N} \mid q \leq \psi e\right\}
$$

for some fixed, but finite $\psi \in \Re_{+}$, where $e$ is the vector of all 1 's. ${ }^{1}$ The cost function is $C \in \mathcal{C}$, where $\mathcal{C}$ is the set of nondecreasing continuously differentiable functions from $\Re_{+}^{N}$ to $\Re_{+}$, with $C(0)=0$. For notational convenience we will denote the partial derivative of $C$ with respect to $q_{i}$ by $\partial_{i} C$ and for $q_{-}, q_{+} \in \Re_{+}^{N}$ define the interval

$$
\left[q_{-}, q_{+}\right]=\left\{p \in \Re_{+}^{N} \mid q_{-} \leq p \leq q_{+}\right\}
$$

A Cost Sharing mechanism, $x$ is given by $x(q ; C) \in \Re_{+}^{N}$ for $q \in Q$ and $C \in \mathcal{C}$. We will make the following standard assumptions on $x$.

Assumption 1. The set of valid Cost Sharing mechanisms, denoted by $x \in$ $\mathcal{X}$, satisfy the following:

1) Budget Balance: $\sum_{i \in N} x_{i}(q ; C)=C(q), \forall q \in Q \quad \forall C \in \mathcal{C}$.

2) Dummy: if $\partial_{i} C \equiv 0$ then $x_{i}(q ; C)=0$.

3) Additivity: $x(q ; C)+x(q ; D)=x(q ; C+D) \forall q \in Q \quad \forall C, D \in \mathcal{C}$.

Budget Balance guarantees that all costs will be recovered, while Dummy requires that agents are not charged for free goods (which have no externalities). The Additivity assumption is nearly universal in the study of Cost Sharing mechanisms (with the notable exceptions of $[9,10,11]$ ). It has several justifications ranging from decentralization to immunity to some accounting issues. (Nonetheless a general theory of nonlinear Cost Sharing mechanisms is an important open problem.)

In addition, we will occasionally require "Null Continuity":

$$
\lim _{q_{i} \rightarrow 0} x\left(q_{i}, q_{-i} ; C\right)=x\left(0_{i}, q_{-i} ; C\right) .
$$

\footnotetext{
${ }^{1}$ Note that we are connecting each $q_{i}$ with a specific agent. As seen from the examples in the Introduction there are instances where we view each $q_{i}$ as associated with a large group of agents. Nonetheless for presentational purposes we will use the term agent to refer to either an agent or a good interchangeably.
} 
This weak axiom requires regularity at zero and removes many complications that can arise under the Scale Invariance axiom described below.

It is convenient to describe Cost Sharing mechanisms with path functions. Let $\gamma(t ; q)$ be a continuous non-decreasing path such that $\gamma(0 ; q)=0$ and $\lim _{t \rightarrow \infty} \gamma(t ; q)=q$. The Cost Sharing mechanism induced by the path function $\gamma$ is given by

$$
x_{i}^{\gamma}(q ; C)=\int_{0}^{\infty} \partial_{i} C(\gamma(t ; q)) d \gamma_{i}(t ; q) .
$$

It is important to note that the parametrization of the of the path does not affect the Cost Sharing mechanism. For example, the Cost Sharing mechanism generated by the path function $\gamma(t ; q)$ is the same as the one generated by $\gamma(\phi(t ; q) ; q)$ where $\phi(t ; q)$ is any continuous scalar function which is nondecreasing in $t$, for fixed $q$ that satisfies $\phi(0 ; q)=0$ and $\lim _{t \rightarrow \infty} \phi(t ; q)=\infty$. Note that the dependence of $\phi$ on $q$ can be completely arbitrary.

For example, the Aumann-Shapley mechanism is given by the well known direct linear path,

$$
\gamma^{A S}(t ; q)=t q \wedge q
$$

where the wedge operator is the componentwise minimum, $(a \wedge b)_{i}=\min \left[a_{i}, b_{i}\right]$, while the Friedman-Moulin mechanism, which is an extension of the Serial Cost mechanism which was defined for homogeneous goods $[12,13]$, is generated by the following path:

$$
\gamma^{F M}(t ; q)=t e \wedge q
$$

where $e$ is the vector of all 1's.

The Shapley-Shubik [14] mechanism can be written as an average over the Random Order mechanisms [15], which are path generated. Let $\pi: N \rightarrow N$ be an ordering of the agents. The Random Order path associated with this ordering is given by

$$
\begin{array}{cll}
\gamma_{i}^{\pi}(t ; q) & 0 & t \leq \pi(i)-1 \\
= & q_{i}(t-\pi(i)+1) & \pi(i)-1<t \leq \pi(i) \\
=q_{i} & & t>\pi(i)
\end{array}
$$

We then write the Random Order mechanism associated with an ordering $\pi \in$ $\Pi$ as $x^{\pi}=x^{\gamma^{\pi}}$ and the Shapley-Shubik mechanism as $x^{S S}=\sum_{\pi \in \Pi} x^{\pi} /|\Pi|$, where $\Pi$ is the set of all orderings. 
We note that any Cost Sharing mechanism can be written as a sum of path mechanisms. This is because the set of Cost Sharing mechanisms is convex so any element of the set can be written as a sum over its extreme points, which are the path generated mechanisms.

Lemma $1([\mathbf{1 6}, \mathbf{1 7}])$. Let $x \in \operatorname{ext}(\mathcal{X})$, the extreme points of the set $\mathcal{X}$. Then $x=x^{\gamma}$ for some path function $\gamma$.

Thus, if we let $\Gamma(q)$ be the set of paths from 0 to $q$ then we can, for any fixed $q$, identify any Cost Sharing mechanism with a probability measure over $\Gamma(q)$ which we denote $\rho(x ; q)$. Similarly, if there are no other constraints, we can identify a full Cost Sharing mechanism with a parameterized set of measures $\rho(x)$ which is a different measure for each $q$. Note that $\rho(x)$ and $\rho(x ; q)$ are not unique as there can be many probability measures representing the same $x .^{2}$

An implicit fact that is very useful, but not explicitly stated in any previous papers is the following. It follows directly from the application of the Riesz representation theorem in the Appendix of [7].

Lemma 2. For any $q \in Q$, if $\gamma_{1}(q), \gamma_{2}(q) \in \Gamma(q)$ and $\gamma_{1}(q) \neq \gamma_{2}(q)$ (there is no reparameterization of the paths that make them identical) then for any $i \in N$ with $q_{i}>0$ there exists some $C \in \mathcal{C}$ such that $x_{i}^{\gamma_{1}}(q ; C) \neq x_{i}^{\gamma_{2}}(q ; C)$.

\section{Axioms and Axiomatizations}

There are four main axioms that we will focus on in this paper. The first is scale invariance which we emphasize refers to relative scale between the different $q_{i}$ 's. It is fundamental when there is no natural comparison scale between agents' demands. First we define Scale Invariance in a manner which will naturally generalize to asymmetric situations.

Definition 1. A Cost Sharing mechanism $x \in \mathcal{X}$ is Scale Invariant ${ }^{3}$ for agent $i$ if for all $q \in Q$ and $\alpha>0$ such that $q_{i}>0$ and $q_{i} / \alpha \leq \psi$,

$$
x(q ; C)=x\left(\frac{q_{i}}{\alpha}, q_{-i} ; \tau_{i}(\alpha) \circ C\right)
$$

\footnotetext{
${ }^{2}$ For example, if two paths intersect each other than one can construct two different paths by joining the initial part (before the intersection) of each path with the latter part of the other path.

${ }^{3}$ Note that we are implicitly assuming that the Cost Sharing Mechanism does not depend on the values of the cost function outside of the set $[0, q]$, a result proved in [7].
} 
where $\tau_{i}(\alpha) \circ C(q)=C\left(\alpha q_{i}, q_{-i}\right)$. A Cost Sharing mechanism $x \in \mathcal{X}$ is Scale Invariant on $A \subseteq N$ if it is Scale Invariant for all $i \in A$.

For later use, we note the following characterization of Scale Invariant Cost Sharing mechanisms, based on the scale invariant paths. However, note that we assumed that $\alpha \neq 0$ in the previous definition and thus scale invariance only connects non-zero demands. Let $\eta(q)=\left\{i \in N \mid q_{i}>0\right\}$ then we say that a path is Scale Invariant on $S \subseteq N$ if there exists a family of continuous and nondecreasing paths, $\sigma\left(t, q_{N \backslash S} ; B\right)$, for all $B \subseteq S$ such that

$$
\gamma(t, q)_{i}=\sigma\left(t, q_{N \backslash S}, \eta(q)\right)_{i} q_{i}
$$

for all $i \in S$ and

$$
\gamma(t, q)_{i}=\sigma\left(t, q_{N \backslash S}, \eta(q)\right)_{i}
$$

for all $i \notin S$. Note that to simplify the presentation, what we are calling a path is actually a set of paths, one for each $q$. Let $\Gamma^{S I}(S)$ be the set of Scale Invariant (families of) paths for $S$.

Lemma 3 ([17]). Let $x \in \mathcal{X}$ be Scale Invariant for $S \subseteq N$. Then there exists some $\rho(x)$ that has full support on $\Gamma^{S I}(S)$ and generates $x$.

Note that the measure is on scale invariant (families of) paths as mentioned above, so is essentially an infinite sum over (sets of) paths. The proof of this lemma is a slight modification of the proof in [17]. For any $i \in S$ and fixed $\eta(q)$ one can take a path decomposition of $x\left(\psi e_{S}, q_{-S} ; \cdot\right)$ and note that a path decomposition for any $x(q ; \cdot)$ can be written by simply extending $\gamma_{i}\left(t ; \psi e_{S}, q_{-S}\right)$ by setting $\gamma_{i}(t ; q)=\gamma_{i}\left(t ; \psi e_{i}, q_{-i}\right) q_{i} / \psi$ for all $i \in S$.

Next we consider the Average Cost Pricing for Homogeneous Goods axiom. In this case instead of cost shares $x_{i}(q ; C)$ we consider the average prices $p_{i}(q ; C)=x_{i}(q ; C) / q_{i}$. In the case where the goods are essentially the same we want these prices to be equal, in order to prevent arbitrage opportunities among agents with infinitesimal demands. This occurs when the cost function is homogeneous, i.e. if $C \in \mathcal{C}^{H}$ then $C(q)=D(|q|)$ for some scalar function $D$ where $|q|=\sum_{i \in N} q_{i}$. We also say the a cost function is homogeneous on a subset $A \subseteq N$ of agents if we can write $C(q)=D\left(\left|q_{A}\right|, q_{-A}\right)$.

Definition 2. A Cost Sharing mechanism $x \in \mathcal{X}$ satisfies Average Cost Pricing for Homogeneous Goods on $A \subseteq N$ if $p_{i}(q ; C)=p_{j}(q ; C)$ for all $i, j \in$ $A$ and $q \in Q$ with $q_{i}, q_{j}>0$ when the cost function $C \in \mathcal{C}$ is homogeneous on $A$ 
For later use, we quote the following axiomatization of the AumannShapley mechanism.

Theorem 1 ([3, 4]). Let $x \in \mathcal{X}$ be a Cost Sharing mechanism that is Scale Invariant and satisfies Average Cost Pricing for Homogeneous Goods on $N$. Then $x \in \mathcal{X}$ is the Aumann-Shapley mechanism.

In the case where each $q_{i}$ is controlled by a single agent, it is important not to have an incentive to overstate demands, which motivates the Demand Monotonicity axiom.

Definition 3. A Cost Sharing mechanism $x \in \mathcal{X}$ is Demand Monotonic on agent $i$ if $x_{i}(q ; C) \leq x_{i}\left(q_{i}^{\prime}, q_{-i} ; C\right)$ for all $q \in Q$ with $q_{i}^{\prime}>q_{i}$. A Cost Sharing mechanism $x \in \mathcal{X}$ is Demand Monotonic on $S \subseteq N$ if it is Demand Monotonic for all $i \in S$.

A fixed path for $S \subseteq N$ can be written as $\gamma(t, q)$ where $\gamma_{S}(t, q)=$ $\hat{\gamma}_{S}\left(t ; q_{-S}\right) e_{S} \wedge q_{S}$ for some function $\hat{\gamma}$ and let $\Gamma^{D M}(S)$ be the set of such paths. Note that as in the definition of Scale Invariant paths, when we refer to a fixed path, we are also referring to the family of paths generated by the fixed path, one for each $q \in Q$.

Lemma 4 ([17]). Let $x \in X$ be Demand Monotonic for $S \subseteq N$. Then there exists some $\rho(x)$ that has full support on $\Gamma^{D M}(S)$.

The proof of this lemma is a slight modification of the proof in [17] which is based on a characterization of Demand Monotonic Cost Sharing mechanisms in [7]. Note that the decomposition of Demand Monotonic Cost Sharing Mechanism as convex combinations of fixed paths relies on our assumption that $q$ is bounded. For example, for unbounded domains it is not possible to write a random order mechanism as a fixed path mechanism.

Lastly we consider an axiom which protects agents from extreme costs imposed by other agents. For example, consider a 2 agent problem where the cost function is $C(q)=\left(q_{1}+q_{2}\right)^{2}$ and the Cost Sharing Mechanism satisfies Average Cost Pricing for Homogeneous Goods (such as the Aumann-Shapley mechanism). Then

$$
x_{1}(q ; C)=q_{1}\left(q_{1}+q_{2}\right)^{2} /\left(q_{1}+q_{2}\right)=q_{1}\left(q_{1}+q_{2}\right)
$$

is strictly increasing in $q_{2}$ and can be arbitrarily large for fixed $q_{1}$. The Upper Bound for Homogeneous Goods axiom prevents this with the tightest uniform bound possible. 
Definition 4. A Cost Sharing mechanism $x \in \mathcal{X}$ satisfies Upper Bound for Homogeneous Goods on $S \subseteq N$ if

$$
x_{i}(q ; C) \leq C\left(q_{i} e\right)
$$

for all $q \in Q$ and $i \in S$ when the cost function $C \in \mathcal{C}$ is homogeneous on $S$.

Next, we quote the following axiomatization of the Friedman-Moulin mechanism.

Theorem 2 ([7]). Let $x \in \mathcal{X}$ be a Cost Sharing mechanism that is Demand Monotonic and satisfies the Upper Bound for Homogeneous Goods on $N$. Then $x \in \mathcal{X}$ is the Friedman-Moulin mechanism.

Finally, we note another useful axiomatization.

Theorem 3 ([7]). Let $x \in \mathcal{X}$ be a Cost Sharing mechanism that is Scale Invariant and Demand Monotonic on $N$. Then $x \in \mathcal{X}$ is a Random Order mechanism [15].

Lastly we note the following impossibility results, which slightly generalize those found in [7].

Theorem 4. There is no Cost Sharing Mechanism satisfying any one of the following pairs of axioms on any subset $S \subseteq N$ with $|S| \geq 2$ :

1) Average Cost Pricing for Homogeneous Goods and Demand Monotonicity.

2) Average Cost Pricing for Homogeneous Goods and Upper Bound for Homogeneous Goods.

3) Scale Invariance and Upper Bound for Homogeneous Goods.

Proof: This result is proven in [7] for the case that $S=N$. To prove it for proper subsets of $N$ one only need consider cost functions for which agents not in $S$ are Dummies. 


\section{Mixing Mechanisms}

We first consider the issue of directly mixing different Cost Sharing mechanisms, where precise Cost Sharing mechanisms are specified on subgroups of the agents. Given a subset of the agents, $A \subseteq N$ we say that the Cost Sharing mechanism reduces to $y$ on $A$ if $x_{i}(q ; C)=y_{i}\left(q_{A} ; D\right)$ for all $i \in A$ when $C(q)=D\left(q_{A}\right)$ for all $q \in Q$. This says that the Cost Sharing mechanism reduces to $y$ when all agents in $N \backslash A$ are Dummies.

We now consider the problem when we want the Cost Sharing mechanism to reduce to two different Cost Sharing mechanisms on different subsets of the agents. For concreteness we consider mixing Aumann-Shapley with Friedman-Moulin, i.e. the mechanism reduces to Aumann-Shapley on $A$ and Friedman-Moulin on $S=N \backslash A$. It is easy to see that such a mechanism exists, by simply combining the path functions for the individual mechanisms:

$$
\gamma(t ; q)=\left(t q_{A} \wedge q_{A}, t e_{S} \wedge q_{S}\right)
$$

Our first result is that the only such Cost Sharing mechanisms are of this type.

Theorem 5. $x \in \mathcal{X}$ is a Cost Sharing mechanism that reduces to the AumannShapley mechanism on $A \subset N$ and Friedman-Moulin on $S=N \backslash A$ such that $|A| \geq 2$ and $|S| \geq 2$, if and only if $x \in \mathcal{X}$ is a convex combination of path generated mechanisms, with paths given by

$$
\gamma(t ; q)=\left(\phi^{A}(t ; q) q_{A} \wedge q_{A}, \phi^{S}(t ; q) e_{S} \wedge q_{S}\right),
$$

where both $\phi^{A}(t ; q)$ and $\phi^{S}(t ; q)$ are continuous nondecreasing functions of $t$ and both

$$
\lim _{t \rightarrow \infty} \phi^{A}(t ; q)=\infty
$$

and

$$
\lim _{t \rightarrow \infty} \phi^{S}(t ; q)=\infty
$$

for all $q \in Q$.

Proof: It is easy to see that if both $\phi^{A}(t ; q)$ and $\phi^{S}(t ; q)$ are continuous nondecreasing functions of $t$ then

$$
\gamma(t ; q)=\left(\phi^{A}(t ; q) q_{A} \wedge q_{A}, \phi^{S}(t ; q) e_{S} \wedge q_{S}\right)
$$


is a valid path and satisfies the basic axioms. Then one can see directly from formula $\left(^{*}\right)$ that if $C(q)$ is independent of $q_{A}$ then the cost shares are 0 for the players in $A$ and the same as the Friedman-Moulin on $S$. Similarly it follows directly that the mechanism projects to the Aumann-Shapley on $A$.

To show the converse consider a Cost Sharing mechanism satisfying the assumptions of the theorem and a fixed $q$. First we recall the following result from [7] (Lemma 3). Any $x \in \mathcal{X}$ can be written as

$$
x_{i}(q ; C)=\int_{[0, q]} \partial_{i} C(p) d \mu_{i}^{q}(p) \quad(* *)
$$

where $\mu_{i}^{q}$ is a non-negative radon measure on $[0, q]$ such that for all $0<a<$ $b<q_{i}$

$$
\mu_{i}^{q}\left(\left\{z \in[0, q] \mid a \leq q_{i} \leq b\right\}\right)=b-a .
$$

Now, fix $q$ and for any $i \in B \subset N$ define $\xi_{B}\left(\mu_{i}^{q}\right)$ to be the projection of $\mu_{i}^{q}$ on $\Re_{+}^{B}$. Formally, for any set $H \subset\left[0, q_{B}\right]$ define

$$
\xi_{B}\left(\mu_{i}^{q}\right)(H)=\mu_{i}^{q}\left(H \times\left[0, q_{N \backslash B}\right]\right) .
$$

Given $i \in B$ note that if $C$ is such that all $j \in N \backslash B$ are Dummy agents then

$$
x_{i}(q ; C)=\int_{[0, q]} \partial_{i} C\left(p_{B}, 0_{N \backslash B}\right) d \pi_{B}\left(\mu_{i}^{q}(p)\right) .
$$

(This is analogous to the argument given in Section 4 of [17].)

Next, let $X$ be the Cost Sharing mechanism satisfying the above assumptions. As shown in [17], $x$ can be approximated (in the Prohorov metric of the underlying measures) by a finite convex combination of path generated methods. In fact, it can be approximated as a convex combination of a fixed number of paths, all with equal weights. Consider the simultaneous approximation, one for each $q$, of $x(q ; \cdot)$ into $r$ equally weighted paths that provide the best approximations.

Consider any such a path, $\gamma(t ; q)$, after this decomposition. From the argument in the previous paragraph, the support of its projection onto $A$ must be the path for the Aumann-Shapley Mechanism. Thus, we can define $\phi^{A}(t ; q)$ by the condition that $\phi^{A}(t ; q) q_{A} \wedge q_{A}=\gamma_{A}(t ; q)$. Similarly, we can define $\phi^{S}(t ; q)$ by the condition that $\phi^{S}(t ; q) e_{S} \wedge q_{S}=\gamma_{S}(t ; q)$. By monotonicity of the path we see that both $\phi^{A}(t ; q)$ and $\phi^{S}(t ; q)$ are continuous nondecreasing functions of $t$. 
To complete the proof let $r \rightarrow \infty$ and note that for each $q$ the approximate mechanism converges to the true one.

It is interesting to note that these mixtures can be extremely unstable. For example, let $\left(\phi^{A}(t ; q), \phi^{S}(t ; q)\right)=(t, \max [0, t-1])$ when all $q_{i}$ are rational and $\left(\phi^{A}(t ; q), \phi^{S}(t ; q)\right)=(t|q|, \max [0, t-1])$ otherwise. It is easy to see that for many reasonable $C \in C$ the cost shares will differ depending on whether $q$ is rational. A simple example arises when $C(q)=\max \left[0,(|q|-1)^{2}\right.$ and $\left|q_{A}\right|=2 / 3$ while $\left|q_{S}\right|=2 / 3$. If all the components of $q_{a}$ are rational, then $\sum_{i \in A} x_{i}=0$ while if any are irrational $\sum_{i \in A} x_{i}=1 / 9 .{ }^{4}$

Even if we eliminate the Cost Sharing mechanisms that are non-differentiable we are still left with an infinite number of possibilities. ${ }^{5}$ (This can be done by requiring that the both $\phi^{A}(t ; q)$ and $\phi^{S}(t ; q)$ be continuous in $\left.q.\right)$

\section{Mixing Axioms}

In this section we explore a subtle distinction. In the previous section we essentially mixed different Cost Sharing mechanisms. In this section we mix the underlying axioms that axiomatize those Cost Sharing mechanisms. The reason that this is nontrivial can be seen by considering the mechanisms analyzed in the previous section. Even though $x \in \mathcal{X}$ reduces to AumannShapley on $A$ and the Aumann-Shapley mechanism is Scale Invariant, $x \in \mathcal{X}$ need not be Scale Invariant on $A$. Additionally even though $x \in \mathcal{X}$ reduces to Friedman-Moulin on $N \backslash A$ it need not be Demand Monotonic on $N \backslash A$. This should be clear from the example of an everywhere discontinuous Cost Sharing mechanism in the previous section, but is true even for many wellbehaved mechanisms.

Theorem 6. For any $A \subset N$ and $S=N \backslash A$, such that $|A| \geq 2$ and $|S| \geq 2$, $x \in \mathcal{X}$ is a Cost Sharing mechanism that satisfies all of the following:

1) $x$ reduces to the Aumann-Shapley mechanism on $A$ and Friedman-Moulin on $S$,

2) $x$ is Scale Invariant on $A$ and Demand Monotonic on $S$,

\footnotetext{
${ }^{4}$ Although the discontinuities only occur on a set of measure 0 , it is easy to modify this example using Cantor sets to get discontinuities on sets of positive measure.

${ }^{5} \mathrm{An}$ anonymous referee has pointed out the amusing example where we mix a Cost Sharing mechanism with itself and similar instability results arise.
} 
if and only if $x \in \mathcal{X}$ is a convex combination of path generated mechanisms, with paths given by

$$
\gamma(t ; q)=\left(\phi^{A}\left(t, \eta\left(q_{A}\right)\right) q_{A}, \phi^{S}\left(t, \eta\left(q_{A}\right)\right) e_{S}\right) \wedge q,
$$

where both $\phi^{A}(t)$ and $\phi^{S}(t)$ are nondecreasing functions of $t$, and both

$$
\begin{aligned}
& \lim _{t \rightarrow \infty} \phi^{A}(t)=\infty, \\
& \lim _{t \rightarrow \infty} \phi^{S}(t)=\infty,
\end{aligned}
$$

and $\eta\left(q_{A}\right)=\left\{i \in A \mid q_{A}>0\right\}$.

Proof: From the previous theorem, we know that all $x$ satisfying (1) can be written as a convex combination of path generated mechanisms with paths of the form

$$
\gamma(t ; q)=\left(\phi^{A}(t ; q) q_{A} \wedge q_{A}, \phi^{S}(t ; q) e_{S} \wedge q_{S}\right),
$$

where both $\phi^{A}(t ; q)$ and $\phi^{S}(t ; q)$ are nondecreasing functions of $t$ and both

$$
\lim _{t \rightarrow \infty} \phi^{A}(t ; q)=\infty
$$

and

$$
\lim _{t \rightarrow \infty} \phi^{S}(t ; q)=\infty
$$

for all $q \in Q$. Assume that $q_{i}>0$ for all $i \in A$. Now, by scale invariance, we can replace every path

$$
\gamma(t ; q)=\left(\phi^{A}(t ; q) q_{A} \wedge q_{A}, \phi^{S}(t ; q) e_{S} \wedge q_{S}\right),
$$

by

$$
\gamma(t ; q)=\left(\phi^{A}\left(t ; q_{S}, e_{A}\right) q_{A} \wedge q_{A}, \phi^{S}\left(t ; q_{S}, e_{A}\right) e_{S} \wedge q_{S}\right),
$$

thus eliminating the dependence of both $\phi^{A}$ and $\phi^{S}$ on $q_{A}$. If some $q_{i}=0$ for $i \in A$ we simply modify this argument, as in the construction of the scale invariant paths to get:

$$
\gamma(t ; q)=\left(\phi^{A}\left(t ; q_{S}, e_{\eta\left(q_{A}\right)}, \eta\left(q_{A}\right)\right) q_{A} \wedge q_{A}, \phi^{S}\left(t ; q_{S}, e_{\eta\left(q_{A}\right)}, \eta\left(q_{A}\right)\right) e_{S} \wedge q_{S}\right) .
$$

Next consider $x(q)$ where $q_{S}=\psi e$ and apply Demand Monotonicity. From the analysis in Appendix B of [7], Demand Monotonicity implies that 
$\mu_{i}^{q}(p)$ must not change (on $\left[0, q_{A}\right]$ ) if $q_{i}$ is increased. Thus for all paths of the form

$$
\gamma(t ; q)=\left(\phi^{A}\left(t ; q_{S}, e_{\eta\left(q_{A}\right)}, \eta\left(q_{A}\right)\right) q_{A} \wedge q_{A}, \phi^{S}\left(t ; q_{S}, e_{\eta\left(q_{A}\right)}, \eta\left(q_{A}\right)\right) e_{S} \wedge q_{S}\right),
$$

we can replace them by

$$
\gamma(t ; q)=\left(\phi^{A}\left(t ; e_{S}, e_{\eta\left(q_{A}\right)}, \eta\left(q_{A}\right)\right) q_{A} \wedge q_{A}, \phi^{S}\left(t ; e_{S}, e_{\eta\left(q_{A}\right)}, \eta\left(q_{A}\right)\right) e_{S} \wedge q_{S}\right)
$$

which leaves them in the form as stated by the theorem, completing the proof.

This allows us to characterize a mixed axiomatic characterization.

Corollary 1. For any $A \subset N$ and $S=N \backslash A$, with $|A| \geq 2$ and $|S| \geq 2$ let $x \in \mathcal{X}$ be a Cost Sharing mechanism that satisfies all of the following:

1) $x$ is Scale Invariant on $A$ and satisfies Average Cost for Homogeneous on A.

2) $x$ is Demand Monotonic on $S$ and satisfies Upper Bound for Homogeneous on $S$.

Then $x \in \mathcal{X}$ is a convex combination of path generated mechanisms, with paths given by

$$
\gamma(t ; q)=\left(\phi^{A}\left(t, \eta\left(q_{A}\right)\right) q_{A}, \phi^{S}\left(t, \eta\left(q_{A}\right)\right) e_{S}\right) \wedge q,
$$

where both $\phi^{A}(t)$ and $\phi^{S}(t)$ are nondecreasing functions of $t$, and both

$$
\begin{aligned}
& \lim _{t \rightarrow \infty} \phi^{A}(t)=\infty, \\
& \lim _{t \rightarrow \infty} \phi^{S}(t)=\infty .
\end{aligned}
$$

There are still infinitely many such mechanisms but they are now continuous, except possibly at $q_{i}=0$ for some $i \in A$, which we could remedy by requiring Null Continuity.

Corollary 2. For any $A \subset N$ and $S=N \backslash A$, let $x \in \mathcal{X}$ be a Cost Sharing mechanism that reduces to the Aumann-Shapley mechanism on $A$ and Friedman-Moulin on $S$. In addition it is Scale Invariant on $A$ and Demand Monotonic on $S$. Then $x(q ; C)$ is continuous in $q$ for any $C \in C$ and $q>0$. 
Proof: To prove this, consider a single path generated mechanism, $x^{\gamma}$, with path of the form

$$
\gamma(t ; q)=\left(\phi^{A}(t) q_{A}, \phi^{S}(t) e_{S}\right) \wedge q .
$$

That $x^{\gamma}$ will be continuous follows directly from the formula for the path generated Cost Sharing mechanisms, the fact that $\partial_{i} C$ is continuous on $[0, q]$ and the continuity of the path $\gamma$ as a function of $q$ which can be seen directly from its formula.

\section{Agreement Points and Unbiasedness}

In this section we characterize a class of interesting mixed Cost Sharing mechanisms. Our first approach is based on the fact that the AumannShapley mechanism and the Friedman-Moulin mechanism agree when players have identical demands. Thus, it seems natural that the mixed mechanism should satisfy this property. However, this is not possible.

Theorem 7. For any $A \subset N$ and $S=N \backslash A$, such that $|A| \geq 2$ and $|S| \geq 2$, there does not exist a Cost Sharing mechanism $x \in \mathcal{X}$ that satisfies all of the following:

1) $x$ reduces to the Aumann-Shapley mechanism on $A$ and Friedman-Moulin on $S$.

2) $x$ is Scale Invariant on $A$ and Demand Monotonic on $S$.

3) $x$ is Null Continuous.

4) For all $\alpha>0$, the point $\alpha e$ is an agreement point, i.e., for all $C \in \mathcal{C}$,

$$
x(\alpha e ; C)=x^{A S}(\alpha e ; C)=x^{F M}(\alpha e ; C) .
$$

Proof: This will follow directly from the next theorem.

Consider a family of mixed mechanisms, the $\alpha$-mixed Cost Sharing mechanisms, $x^{\alpha}$, which reduce to the Aumann-Shapley mechanism or FriedmanMoulin mechanism for the symmetric demand $q=\alpha e$, where $x^{\alpha}$ is generated by the path:

$$
\gamma(t ; q)=\left(t q_{A},(t / \alpha) e_{S}\right) \wedge q
$$

These are characterized by this property. 
Theorem 8. For any $A \subset N$ and $S=N \backslash A$, such that $|A| \geq 2$ and $|S| \geq 2$, given $\alpha>0$ there exists a unique Cost Sharing mechanism, the $\alpha$-mixed Cost Sharing mechanism, $x^{\alpha} \in \mathcal{X}$ that satisfies all of the following:

1) $x$ reduces to the Aumann-Shapley mechanism on A and Friedman-Moulin on $S$.

2) $x$ is Scale Invariant on $A$ and Demand Monotonic on $S$.

3) $x$ is Null Continuous.

4) The point $\alpha e$ is an agreement point, for all $C \in \mathcal{C}$,

$$
x(\alpha e ; C)=x^{A S}(\alpha e ; C)=x^{F M}(\alpha e ; C) .
$$

Proof: From the previous theorem we know that we only need to consider convex combinations of paths of the form

$$
\gamma(t ; q)=\left(\phi^{A}\left(t, \eta\left(q_{A}\right)\right) q_{A}, \phi^{S}\left(t, \eta\left(q_{A}\right)\right) e_{S}\right) \wedge q .
$$

In addition by Null Continuity we can assume that $q>0$ and thus ignore the dependence of $\phi^{A, S}$ on $\eta(q)$.

First consider $q=\alpha e$ and note that the Cost Sharing mechanism must be the Aumann-Shapley mechanism which in this case is the same as the Friedman-Moulin mechanism and is generated by the path which is a line from the origin to $\alpha e$. By scale invariance this implies that for any $q>0$ such that $q_{S}=\alpha e_{S}$ the mechanism $x(q, \cdot)$ is generated by the path

$$
\gamma(t ; q)=\left(t q_{A}, t \alpha e_{S}\right) \wedge q
$$

Comparing this with the general form for these paths

$$
\gamma(t ; q)=\left(\phi^{A}(t) q_{A}, \phi^{S}(t) e_{S}\right) \wedge q
$$

we see that $\phi^{A}(t)=t$ for $t<1$ and $\phi^{A}(t)$ is not relevant for $t>1$, so we need only consider paths of the form

$$
\gamma(t ; q)=\left(t q_{A}, \phi^{S}(t) e_{S}\right) \wedge q
$$

Next, by demand monotonicity, the "projection" of the paths must be unchanged on $[0, q]$ if $q_{S} \leq \alpha e_{S}$ and unchanged on $\left[0,\left(q_{A}, \alpha e_{S}\right)\right]$ if $q_{S}>\alpha e_{S}$. This implies that $\phi^{S}\left(t, q_{S}\right)=t$ for $t \leq 1$ but the generated Cost Sharing mechanism does not depend on $\phi^{S}\left(t, q_{S}\right)$ for $t>1$, since $\gamma_{A}(t ; q)$ is constant in that case. 
Thus, every path in the decomposition of $x$ is equivalent to the path that generates the $\alpha$-mixed Cost Sharing mechanism, completing the proof.

The Cost Sharing mechanisms defined in the previous theorem are well defined for limiting values of $\alpha$. For example, when $\alpha=0$ the Cost Sharing mechanisms converge to 0 -mixed Cost Sharing mechanism, $x^{\alpha=0}$, in which we first apply the Friedman-Moulin mechanism among the agents in set $S$, setting $q_{A}=0$, then apply the Aumann-Shapley mechanism to the remaining cost function $C\left(q_{A}, q_{S}\right)-C\left(0_{A}, q_{S}\right)$, while we can define the $\infty$-mixed Cost Sharing mechanism, $x^{\alpha=\infty}$, to be the reverse procedure where we first allocate cost shares among the agents in $A$ then the residual cost using the Friedman-Moulin mechanism among the agents in $S$. Note that this procedure generalizes to any mixture of mechanisms and can generate simple, but extremely asymmetric, cost allocations; however the "mixed-mixed" Cost Sharing mechanism which is constructed by $x=(1 / 2) x^{\alpha=0}+(1 / 2) x^{\alpha=\infty}$, is an interesting mixture which has aspects of the Shapley-Shubik mechanism on top of a mixture of the Aumann-Shapley mechanism and the FriedmanMoulin mechanism.

In fact, we can characterize the "mixed-mixed" Cost Sharing mechanism by a very weak symmetry requirement. We say that a Cost Sharing mechanism is Unbiased if the cost shares are equal when everything else is equal. We define a cost function to be symmetric if it is unchanged by any permutation of its arguments.

Definition 5. A Cost Sharing mechanism is Unbiased if for all $\alpha>0$ and all symmetric $C \in \mathcal{C}$,

$$
x(\alpha e, C)=C(\alpha e) / n .
$$

Note that this differs from the previous requirement in that we only consider symmetric cost functions. It is easy to see that "mixed-mixed" Cost Sharing mechanism is unbiased. In fact, it is the only unbiased mixture.

Theorem 9. For any $A \subset N$ and $S=N \backslash A$ such that $|A| \geq 2$ and $|S| \geq 2$, there is a unique Cost Sharing mechanism $x \in \mathcal{X}$ that satisfies all of the following:

1) $x$ reduces to the Aumann-Shapley mechanism on $A$ and Friedman-Moulin on $S$.

2) $x$ is Scale Invariant on $A$ and Demand Monotonic on $S=N \backslash A$, when 
$1<|A|<n$.

3) $x$ is Null Continuous 6 .

4) $x$ is Unbiased.

Proof: (Sketch) First, note that it is easy to check that the mechanism described above satisfies all the requirements, so we only need to prove uniqueness. Next, note that for the mechanism described above all paths are contained on the four faces defined by $p_{A}=0, p_{A}=q_{A}, p_{S}=0$ and $p_{S}=q_{S}$. Our goal is to show that this must be true of any $x$ satisfying the assumptions of the Theorem. To prove this for any $x$ satisfying the assumptions of this theorem we assume, for contradiction, that there exists some $\hat{q}>0$ and some $\hat{p}<\hat{q}$ such that for some $\hat{i} \in A$ and $\hat{j} \in S, \hat{p}_{\hat{i}} \in\left(0, \hat{q}_{\hat{i}}\right)$ and $\hat{p}_{\hat{j}} \in\left(0, \hat{q}_{\hat{j}}\right)$, the set of paths passing through any neighborhood of $p$ has nonzero measure. By Demand Monotonicity we can assume that $\hat{q}_{S}=\bar{q}_{S}$, where $\bar{q}=\psi e$, since that does not alter the paths at $\hat{p}$ and by Scale Invariance we can assume, by rescaling, that $\hat{q}_{A}=\bar{q}_{A}$. Thus, we consider $x(\bar{q}, \cdot)$ and the point $\hat{p}$. Also assume that $\hat{i}$ maximizes $\hat{p}_{i}$ for $i \in A$ and $\hat{j}$ maximizes $\hat{p}_{j}$ for $j \in S$.

We first consider the case where $\hat{p}_{\hat{i}}>\hat{p}_{\hat{j}}$. Consider a continuously differentiable monotonic function $g_{\epsilon}(q)$, for $0<\epsilon<1 / 4$, which is 0 if $q \leq 1-\epsilon$ and 1 if any $q_{i}>1$ for some $i \in N$ and define $h_{\epsilon, a, b}(q)=g_{\epsilon}\left(q_{A} / a, q_{S} / b\right)$. Note that as $\epsilon \rightarrow 0$ this function converges to a step function on the largest $q_{i}$.

Set $a=\hat{p}_{\hat{j}}-\delta$ and and define $\alpha=\left(\hat{p}_{\hat{j}}-\delta\right) /\left(\hat{p}_{i} h+\delta\right)$ for $\delta>0$ sufficiently small. By unbiasedness,

$$
x_{i}\left(\alpha \bar{q} ; h_{\epsilon, a, a}\right)=1 / n,
$$

for all $i \in N$. By demand monotonicity

$$
x_{i}\left(\alpha \bar{q}_{A}, \bar{q}_{S} ; h_{\epsilon, a, a}\right)=1 / n,
$$

for all $i \in N$. Then by scale invariance

$$
x_{i}\left(\bar{q} ; h_{\epsilon, a / \alpha, a}\right)=1 / n,
$$

for all $i \in N$. However,

$$
x_{i}\left(\bar{q} ; h_{\epsilon, a / \alpha, a / \alpha}\right)=1 / n,
$$

\footnotetext{
${ }^{6}$ Null Continuity is used here to simplify the statement of the Theorem. Alternatively, one could impose a stronger version of Unbiasedness which rely only on the players with nonzero demands.
} 
by Unbiasedness. Since an addition nonzero measure of paths passes through $h_{\epsilon, a / \alpha, a / \alpha}$ but doesn't pass through $h_{\epsilon, a / \alpha, a}$ we see that

$$
x_{i}\left(\bar{q} ; h_{\epsilon, a / \alpha, a / \alpha}\right)>x_{i}\left(\bar{q} ; h_{\epsilon, a / \alpha, a}\right),
$$

yielding a contradiction.

One can apply the same argument when $\hat{p}_{\hat{i}}<\hat{p}_{\hat{j}}$ by using a function which converges to a step function on the smallest $q_{i}$ instead of the largest $q_{i}$. Thus we see that the paths in the Cost Sharing mechanism lie on the faces described above. It is straightforward, although tedious, to show that this fact, combined with the assumptions of the theorem lead to the mixed-mixed Cost Sharing mechanism.

\section{Multiple Paths and Internal Symmetry}

In this section we consider mixtures of the Aumann-Shapley mechanism and the Shapley-Shubik mechanism to highlight some symmetry issues which arise from the fact the the Shapley-Shubik mechanism is not generated by a single path. Analogously to the previous section assume that we want the Cost Sharing mechanism to reduce to the Aumann-Shapley mechanism on $A \subset N$ and the Shapley-Shubik mechanism on $S=N \backslash A$, assuming that $|A| \geq 2$ and $|S| \geq 2$. Clearly, there exist Cost Sharing mechanisms $x$ which satisfy these requirements. However, even though the AumannShapley mechanism and the Shapley-Shubik mechanism are both Scale Invariant the mixture mechanism need not be.

Theorem 10. For any $A \subset N$ and $S=N \backslash A$ such that $|A| \geq 2$ and $|S| \geq 2$, there exist Cost Sharing mechanisms $x \in \mathcal{X}$ that satisfy the following:

1) $x$ reduces to the Aumann-Shapley mechanism on A and the Shapley-Shubik mechanism on $S$.

2) $x$ is not scale invariant for any $i \in N$.

Proof: Let $\gamma^{A S}\left(t ; q_{A}\right)$ be the path that generates the Cost Sharing mechanism for the Aumann-Shapley mechanism on $A$ and $\gamma^{\pi}\left(t ; q_{S}\right)$ be the path that generates the Cost Sharing mechanism for a Random Order mechanism on $S$. Then simply construct

$$
\gamma^{A S \pi}(t ; q)=\left(\gamma^{A S}\left(t ; q_{A}\right), \gamma^{\pi}\left(t\left|q_{A}\right| ; q_{S}\right)\right)
$$


and construct $x$ as the average over all such path generated mechanisms (where the average is over $\pi \in \Pi$ ). It is easy to check that this Cost Sharing mechanism is not Scale Invariant, since for all $\pi \in \Pi$ the Cost Sharing mechanism $x^{\gamma^{A S \pi}}$ is not Scale Invariant, since the underlying path is not Scale Invariant. Since the scale invariant paths form a basis for the scale invariant mechanisms [17] this completes the proof.

One interesting difference in combining the Aumann-Shapley mechanism with the Shapley-Shubik mechanism from the previous section (combining the Aumann-Shapley mechanism with the Friedman-Moulin mechanism) arises from the fact that the Shapley-Shubik mechanism is not generated by a single path. This allows more freedom in the possible extensions and shows that many basic properties of the Cost Sharing mechanisms can be lost by asymmetric axiomatizations. In particular, even though both the AumannShapley mechanism and the Shapley-Shubik mechanism are symmetric, one can construct extensions without symmetry, even among agents in the same set.

To formulate this define, for any pair of agents, $i, j \in N$, the exchange operator $\tau_{i, j}$ such that $\tau_{i, j}(q)_{j}=q_{i}$ and $\tau_{i, j}(q)_{i}=q_{j}$, while $\tau_{i, j}(q)_{k}=q_{k}$ for $k \neq i, j$. Its action on functions is defined similarly, $\tau_{i, j} \circ C(q)=C\left(\tau_{i, j}(q)\right)$.

Definition 6. A Cost Sharing mechanism is Symmetric with respect to $i, j \in$ $N$ if for all $q \in Q$, all $C \in \mathcal{C}$ and all $i \in N$,

$$
x(q, C)=\tau_{i, j} x\left(\tau_{i, j}(q), \tau_{i, j} \circ C\right) .
$$

It is Symmetric with respect to $B \subseteq N$ if it is Symmetric with respect to all pairs $i, j \in B$.

Thus, in our formulation where $x$ reduces to the Aumann-Shapley mechanism on $A$ and the Shapley-Shubik mechanism on $S$ one might expect $x$ to be Symmetric with respect to $A$ and also Symmetric with respect to $S$ since the Aumann-Shapley mechanism and the Shapley-Shubik mechanism are both Symmetric on all agents. However, this need not be true, even when we mix axiomatizations.

Theorem 11. For any $A \subset N$ and $S=N \backslash A$ such that $|A| \geq 2$ and $|S| \geq 2$, there exist Cost Sharing mechanisms $x \in \mathcal{X}$ that satisfy the following:

1) $x$ reduces to the Aumann-Shapley mechanism on A and the Shapley-Shubik 
mechanism on $S$.

2) $x$ is Scale Invariant on all of $N$ and Demand Monotonic on $S$.

3) $x$ is not Symmetric among some pair $i, j \in S$.

Proof: In order to construct such a Cost Sharing mechanism one need only construct paths that are not symmetric with respect to the underlying orderings. Pick some $i, j \in S$ and for all $\pi \in \Pi$ define

$$
\gamma^{A S \pi}(t ; q)=\left(\gamma^{A S}\left(t ; q_{S}\right), \gamma^{\pi}\left(t ; q_{A}\right)\right)
$$

if $\pi(i)<\pi(j)$ and

$$
\gamma^{A S \pi}(t ; q)=\left(\gamma^{A S}\left(t ; q_{S}\right), \gamma^{\pi}\left(2 t ; q_{A}\right)\right)
$$

otherwise. Let $x$ be the average over all the path generated mechanisms $x^{\gamma^{A S \pi}}$. Choose some $k \in S$ and consider a cost function where all players besides $i, j, k$ are dummies. To calculate $x(q ; C)$ we note that the Cost Sharing mechanism where all other players are dummies is equivalent to that for which we assume there are only those 3 players. In that case, there are two paths in the Cost Sharing mechanism, one in which $i$ precedes $j$ in the ShapleyShubik mechanism and one in which $j$ precedes $i$ in the SSM. Setting $q=e$ and $C(p)=\left(p_{i}+p_{j}+p_{k}\right)^{2}$ and we can compute the cost shares for the first path to be $(2,5,2)$, since it divides the cost on $q_{i}=1, q_{j}=0, q_{k}=1$ evenly among $i, k$ and allocates the remaining cost to $j$; however, the shares differ for the other path, since it first allocates the Aumann-Shapley mechanism costs for $q_{i}=0, q_{j}=1 / 2, q_{k}=1$ to $j, k$ then the excess cost for $q_{i}=0, q_{j}=1, q_{k}=1$ to $j$ then the excess cost for $q_{i}=1, q_{j}=1, q_{k}=1$ to $i$, which yields $(5,5 / 2,3 / 2)$. Taking the average of these two gives $(7 / 2,15 / 4,7 / 8)$ which is not symmetric between $i$ and $j$.

Note that one can extend the proof to construct Cost Sharing mechanisms which are not symmetric for every pair $i, j \in N$. Also, this asymmetry arises because of the multiple paths in the Shapley-Shubik mechanism, as can be seen from the construction used in the proof and these issues can not arise when only path generated Cost Sharing mechanisms are involved. For example, it is easy to show the following:

Corollary 3. For any $A \subset N$ and $S=N \backslash A$ such that $|A| \geq 2$ and $|S| \geq 2$, let $x \in \mathcal{X}$ be a Cost Sharing mechanism that satisfies all of the following: 
1) $x$ is Scale Invariant and satisfies Average Cost for Homogeneous on A.

2) $x$ is Demand Monotonic and satisfies Upper Bound for Homogeneous on S.

Then $x$ is Symmetric among any pair $i, j \in A$ and any pair $i, j \in S$.

Proof: From Theorem 6 we know that any such Cost Sharing mechanism is constructed from paths of the form:

$$
\gamma(t ; q)=\left(\phi^{A}(t) q_{A}, \phi^{S}(t) e_{S}\right) \wedge q .
$$

Thus, it is clear from the symmetry of the path that the induced Cost Sharing mechanism will by also have the required symmetry and any combination of such symmetric Cost Sharing mechanisms will have the required symmetry.

There are two natural, but perhaps unfair, mixtures that are interesting. These are the ordered mixtures. The first is $x^{A S, S S}$ in which we first allocate costs among the agents in $A$ by $x^{A S}\left(q_{A} ; C_{A}\right)$ then allocate the remainder among the agents in $S$ by computing $x^{S S}\left(q_{S} ; C_{S}\right)$ where $C_{A}\left(q_{A}\right)=C\left(q_{A}, 0_{S}\right)$ and $C_{S}\left(q_{S}\right)=C\left(q_{A}, q_{S}\right)$. Reversing the order of allocations yields $x^{A S, S S}$. These have simple axiomatizations.

Theorem 12. For any $A \subset N$ and $S=N \backslash A$, such that $|A| \geq 2$ and $|S| \geq 2$, let $x \in \mathcal{X}$ be a Cost Sharing mechanism such that $x$ reduces to the Aumann-Shapley mechanism on $A$ and Shapley-Shubik on $S$.

1) If $x_{A}(q ; C)$ is independent of $q_{S}$ for all $q_{A} \in Q_{A}$ and $C \in \mathcal{C}$ then $x=$ $x^{A S, S S}$.

2) If $x_{S}(q ; C)$ is independent of $q_{A}$ for all $q_{S} \in Q_{S}$ and $C \in \mathcal{C}$ then $x=x^{S S, A S}$.

However, there is a more interesting mechanism which fits in with the spirit of the Shapley-Shubik mechanism. To define it, consider the path

$$
\gamma^{i, \pi}(t ; q)=\left(\gamma_{A}^{A S}\left(\phi^{A}(t), q_{A}\right), \gamma_{S}^{\pi}\left(\phi^{A}(t), q_{A}\right)\right),
$$

where $\phi^{A}(t)$ is 0 for $t<i, 1$ for $t>i+1$ and $t-i$ otherwise while $\phi^{B}(t)$ is $t$ for $t<i, t-1$ for $t>i+1$ and $i$ otherwise. (This path follows the Random Order path up to agent $i$, then follows the Aumann-Shapley path for the agents in $i$ then returns to the Random Order path.) Define $x^{i, \pi}$ to 
be generated by the path $\gamma^{i, \pi}$. Now we define the Cost Sharing mechanism that mixes all of the $x^{i, \pi}$, the SS-mixed Cost Sharing mechanism:

$$
x^{A S S m}=\frac{1}{|\Pi||S|} \sum_{\pi \in \Pi} \sum_{i \in S} x^{i, \pi} .
$$

Theorem 13. For any $A \subset N$ and $S=N \backslash A$ such that $|A| \geq 2$ and $|S| \geq 2$, the SS-mixed Cost Sharing mechanism is the unique $x \in \mathcal{X}$ such that:

1) $x$ reduces to the Aumann-Shapley mechanism on $A \subset N$ and ShapleyShubik on $S$.

2) $x$ is Symmetric among any pair $i, j \in A$ and any pair $i, j \in S$.

3) For any $i \in A$ the mechanism reduces to the Shapley-Shubik mechanism on $S \bigcup\{i\}$, i.e., $x_{S}\left(0_{A \backslash\{i\}}, q_{i}, q_{S} ; C\right)$ is the Shapley-Shubik mechanism.

Proof: Projecting $\gamma^{j, \pi}$ onto $\{i\} \bigcup S$ for $i \in A$ yields a Random Order path for $\{i\} \bigcup S$ and thus $x$ projects to the Shapley-Shubik mechanism on $\{i\} \bigcup S$ and clearly satisfies the other requirements. To show uniqueness, consider $q$ such that $q_{A}=e_{A} /|A|$ and $q_{S}=e_{S}$. Next consider some $i \in A$ and $j \in S$. This projection of the Cost Sharing mechanism onto $i, j$ is the Shapley-Shubik mechanism, and thus has support only on the edges of the unit square. Also, if $j, k \in S$ then the projection of the Cost Sharing mechanism onto $j, k$ has support only on the diagonal line from $(0,0)$ to $(1,1)$. Combining these shows that the projection onto $\{i\} \bigcup S$ is the sum of two paths, $\left(t \wedge 1, \max (t-1,0) e_{S} \wedge e_{S}\right)$ and $\left(\max (t-1,0) \wedge 1, t e_{S} \wedge e_{S}\right)$. Thus, the support of the Cost Sharing mechanism must have the same support as $x^{A S S m}$ and the fact that the underlying measures are identical follow from symmetry and budget balance.

\section{Discussion}

We have constructed and characterized several interesting mixed Cost Sharing mechanisms and pointed out several subtleties in the analysis of such methods. Nonetheless, our analysis has only touched the surface of this topic of mixed axiomatizations and thus there are many new directions for research and open problems in this area. Many of these problems are amenable to the techniques and analysis used in this paper. One interesting goal is to create a full constructive theory of mixed axiomatizations: given some set valued axioms, such as Scale Invariance, which must hold for agents 
in $A \subseteq N$ while Demand Monotonicity must hold for agents in $B \subseteq N$ and some other axiom that must hold for agents in $E \subseteq N$ where $A, B, E$ are arbitrary, construct a Cost Sharing mechanism that satisfies all of them or show that none exist.

Another direction can be driven by applications. In addition to the motivating examples discussed in the introduction, other examples merit analysis. For example, when sharing water resources [18] the necessary constraints may depend on physical constraint - which farmers can easily share trade water, while similar considerations apply when allocating transmission costs [19].

Lastly, one can consider problems where agents have multidimensional demands. The characterization of cost sharing methods for these problems has been sparse, with the notable exception of [20]. One can apply some of the techniques from this paper to these problems by asymmetric axiomatizations that prevent arbitrage opportunities among different goods demanded by the same agent.

Acknowledgment. I thank Van Kolpin, Rich Mclean, Herve Moulin, Anne van den Nouweland, Yves Sprumont and two anonymous referees for helpful conversations and suggestions and the University of California at Berkeley for its hospitality during much of this work. This research has been supported in part by the NSF under grant CDI-0835706.

\section{References}

[1] L. Shapley, A value for n-person games, in: H. Kuhn, A. Tucker (Eds.), Contributions to the theory of games, Princeton University Press, Princeton, NJ, 1953.

[2] R. J. Aumann, L. S. Shapley, Values of Non-Atomic Games, Princeton University Press, Princeton, N.J., 1974.

[3] L. Billera, D. Heath, Allocation of shared costs: A set of axioms yielding a unique procedure, Mathematics of Operations Research 7 (1) (1982) $32-39$.

[4] L. Mirman, Y. Tauman, Demand compatible equitable cost sharing prices, Mathematics of Operations Research 7 (1) (1982) 40-56. 
[5] H. Moulin, Axiomatic cost and surplus-sharing, in: Arrow, Sen, Suzumura (Eds.), Handbook of Social Choice and Welfare, North-Holland, New York, 2002.

[6] H. Moulin, On additive methods to share joint costs, Japanese Economic Review 4 (1995) 303-332.

[7] E. J. Friedman, H. Moulin, Three methods to share joint costs, Journal of Economic Theory 87 (2) (1999) 275-312.

[8] L. Billera, D. H. andJ. Raanan, Internal telephone billing rates: a novel application of non atomic game theory, Operations Research 26 (1978) 956-65.

[9] Y. Sprumont, Ordinal cost sharing, Journal of Economic Theory 81 (1) (1998) 126-62.

[10] M. Koster, S. Tijs, P. Borm, Serial cost sharing methods for multicommodity situations, Mathematical Social Sciences 36 (3) (1998) 229242 .

[11] M. Koster, The Moulin-Shenker rule, Social Choice and Welfare 29 (2) (2007) 271-293.

[12] H. Moulin, S. Shenker, Serial cost sharing, Econometrica 60 (1992) 10091037.

[13] H. Moulin, S. Shenker, Average cost pricing versus serial cost sharing: An axiomatic comparison, Journal of Economic Theory 64 (1) (1992) $178-201$.

[14] M. Shubik, Incentives, decentralized control, the assignment of joint costs and internal pricing, Management Science 8 (3) (1962) 325-343.

[15] R. Weber, Probabilistic values for games, in: A. Roth (Ed.), The Shapley Value, Cambridge University Press, 1988.

[16] O. Haimanko, Value theory without symmetry, International Journal of Game Theory 29.

[17] E. J. Friedman, Paths and consistency in additive cost sharing, International Journal of Game Theory 32 (4) (2004) 501 - 518. 
[18] D. Aadland, V. Kolpin, Shared irrigation costs: an empirical and axiomatic analysis, Mathematical Social Sciences 35 (2) (1998) 203-218.

[19] M. Bockarjova, M. Zima, G. Andersson, On allocation of the transmission network losses using game theory, in: Proceedings of the 5th International Conference on European Electricity Market, IEEE, 2008, pp. 1-6.

[20] V. Kolpin, Multi-Product Serial Cost Sharing: An Incompatibility with the Additivity Axiom, Journal of Economic Theory 69 (1) (1996) 227233. 